
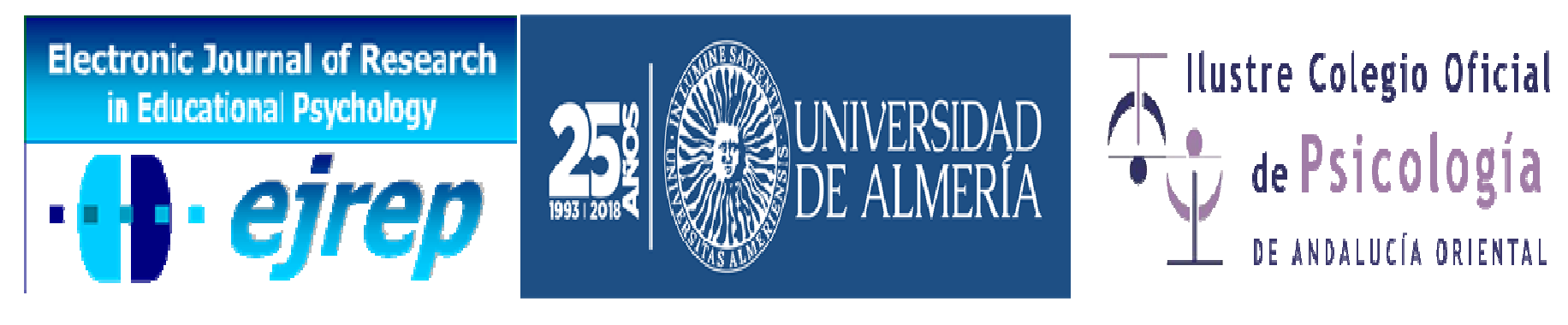

\title{
Actitudes hacia la inclusión de universitarios con discapacidad: Desarrollo y validación de una escala basada en la Teoría de la Conducta Planificada
}

\author{
Eva María Fernández Faúndez ${ }^{1}$
}
${ }^{1}$ Facultad de Educación y Trabajo Social, Universidad de Valladolid, Valladolid

\section{España}

Correspondencia: Eva María Fernández Faúndez, Departamento de Psicología, Facultad de Educación y Trabajo Social, Campus Miguel Delibes, Paseo de Belén 1. CP 47011 Valladolid. España. E-mail: evamfdez@yahoo.es 


\section{Resumen}

Introducción. El foco de estudio de nuestra investigación se centra en el análisis de las actitudes y la predicción del comportamiento de los estudiantes universitarios hacia la inclusión del alumnado con discapacidad en la universidad. La evaluación de las actitudes y la predicción del comportamiento son complejas pero es fundamental por el impacto determinante que tienen sobre la participación del estudiante con discapacidad en el ámbito socio-educativo. Por tanto, para dicho fin se ha desarrollado y validado una escala basada en el marco teórico del modelo de la Teoría de la Conducta Planificada (TPB); uno de los modelos que aporta una visión metodológica más completa para explicar y predecir el comportamiento.

Método. La muestra estuvo formada por 1044 participantes, de los cuáles 623 eran estudiantes de universidades españolas y 421 de universidades argentinas, recogidos con el instrumento diseñado según la $T P B$. Se comprobó la validez y fiabilidad de este instrumento por medio del Análisis Factorial Confirmatorio, procedimiento encuadrado dentro de los modelos de ecuaciones estructurales SEM (Structural Equation Modeling).

Resultados. Los resultados obtenidos en cuanto a la fiabilidad y validez del instrumento confirman la adecuación de la bondad de ajuste, consistencia interna, validez discriminante y validez de criterio. Por otro lado, se observa que en términos generales las respuestas del alumnado sin discapacidad hacia la inclusión del alumnado con discapacidad en la universidad son positivas.

Discusión y conclusiones. A pesar de que existen investigaciones realizadas en el ámbito universitario que analizan las actitudes hacia las personas con discapacidad, son escasas las que emplean el modelo de la Teoría de la Conducta Planificada con suficientes evidencias de fiabilidad y validez. En este sentido, presentamos una escala psicométricamente validada cuyo objetivo es el de evaluar las actitudes y la intención del alumando universitario hacia la inclusión del alumnado con discapacidad en la universidad. De este modo, podremos desarrollar intervenciones para mejorar la inclusión del alumnado con discapacidad en la universidad.

Palabras Clave: actitudes hacia la inclusión, alumnado con discapacidad, educación superior, Teoría de la Conducta Planificada. 


\section{Abstract}

Introduction. The present study is focused on the analysis of attitudes and behavior prediction of higher education students towards the inclusion of students with disabilities at the university. The assessment of attitudes and behavior prediction are complex but it is essential because they have a decisive impact on student's participation in the socio-educational area. For this purpose, we have developed and validated a scale based on the theoretical framework of the Theory of Planned Behavior (TPB); one of the available models that provides a complete methodological solution to explain and predict behavior.

Method. The sample consisted of 1044 participants. 623 were students belonging to Spanish universities and 421 from Argentinian ones. Data was collected using the designed scale inaccordance with TPB. The validity and reliability of the instrument is verified through Confirmatory Factor Analysis, a procedure framed within the well-known Structural Equation Models (SEM).

Results. The results obtained are presented in terms of the reliability and validity of the instrument, which confirm that the model shows indexes of goodness of fit, internal consistency, discriminant validity and criterion-related validity test adequate. On the other hand, it can be observed that in general terms the responses of students without disabilities towards inclusion of students with disabilities in the university are positive.

Discussion and Conclusion. Despite the fact that there are researches in the university that analyze attitudes toward people with disabilities, there are few that use the model of the Theory of Planned Bahavior with evidence of reliability and validity suitable. In this sense, we present a psychometrically validated scale whose objective is to evaluate the attitudes and intention of higher students towards the inclusion of students with disabilities in the university. Thus, we can develop interventions to improve the inclusion of students with disabilities in the university.

Keywords: Attitudes toward inclusion, students with disabilities, higher education, Theory of Planned Behavior. 


\section{Introducción}

Existe un número creciente de estudiantes con discapacidad que desean formar parte del mundo universitario (Konur, 2006), pero en ocasiones encontramos que la universidad es una de las instituciones más excluyentes para el ingreso y la permanencia de estos estudiantes (Moreno, Rodríguez, Saldaña, \& Aguilera, 2006; Suriá, 2011). La Convención de los Derechos de las Personas con Discapacidad, en concreto en el Artículo 24 (ONU, 2006, p. 20), expone que "Los Estados Partes asegurarán que las personas con discapacidad tengan acceso general a la Educación Superior (...) en igualdad de condiciones con las demás”, por lo que es un derecho de las personas con discapacidad sobre el que deben responder las universidades. Este mismo documento destaca que la discapacidad es "Un concepto que evoluciona y que resulta de la interacción entre las personas con deficiencias y las barreras debidas a la actitud y al entorno que evitan su participación plena y efectiva en la sociedad, en igualdad de condiciones con las demás" (ONU, 2006, p. 1).

De este modo, entendemos la inclusión de las personas con discapacidad como el proceso de incrementar y mantener su participación como cualquier otra persona en la sociedad, escuela o comunidad de forma simultánea, procurando disminuir y eliminar todo tipo de procesos que lleven a su exclusión (Booth, 1996). Asimismo, la perspectiva del modelo social de la discapacidad (Barnes, \& Mercer, 2004) que explica que ésta es una forma de opresión social en la que se aboga por la inclusión como parte de las interrelaciones en la sociedad (Barton, 2009) y la exclusión es un problema de justicia social y equidad. Por tanto, la inclusión implicaría la desaparición de toda forma de discriminación, así como la determinación de qué necesidades deben ser cambiadas y cómo (Ainscow, 1999). La discriminación se debe en gran medida al grado de conocimiento que tiene la sociedad en torno a la discapacidad, que es muy limitado y a su vez esta falta de información está vinculada a las actitudes negativas y a las creencias estigmatizantes (Arias \& Morentin, 2003; Hatton, Akram, Robertson, Shah, \& Emerson, 2003). De esta forma, los esfuerzos por conseguir la integración, la independencia y la autodeterminación de las personas con discapacidad encuentran serias resistencias a través de estas actitudes negativas y creencias estigmatizantes (Ali, Strydom, Hassiotis, Williams, \& King, 2008). Por tanto es fundamental detectar estas actitudes negativas para poder realizar intervenciones destinadas al cambio de las mismas. 
Las actitudes hacia las personas con discapacidad han sido medidas a lo largo de la historia con diferentes instrumentos. Esto es debido a que el concepto es complejo y las diversas definiciones sobre el mismo han determinado diferentes modelos explicativos derivados de esta falta de consenso en su delimitación conceptual. Este problema teórico que surge a cerca de cómo definir las actitudes, se hace extensivo a la metodología y, por tanto, a la evaluación (Arias, Verdugo, Gómez \& Arias, 2013). Estos métodos de evaluación han experimentado cambios que van desde la utilización de instrumentos subjetivos, informales y normalmente carentes de validez psicométrica, a instrumentos más objetivos, cuidadosamente planificados y desarrollados que proporcionan datos susceptibles de tratamiento matemático, apoyados en potentes bases metodológicas y sometidas generalmente a análisis multivariados, como explican Verdugo, Arias y Jenaro, (1994) y Verdugo, Jenaro y Arias, (1995). Algunos ejemplos de los instrumentos más utilizados diseñados para medir las actitudes hacia las personas con discapacidad son: Attitude Toward Disabled Persons Scale (ATDP) de Yuker y Block, (1986); Scale of Attitudes Towards Disabled Persons (SADP) de Antonak, (1982) у Escala de Actitudes hacia las Personas con Discapacidad, Forma G, Verdugo et al., (1994) en el contexto Latinoamericano, entre otros.

Aunque existen diversos instrumentos para medir las actitudes hacia las personas con discapacidad, éstos suelen estar destinados al estudio de etapas anteriores a la universidad y a otros agentes implicados. La importancia que tiene el estudio de las actitudes en el contexto universitario es porque cada vez más estudiantes con discapacidad acceden a este contexto y debemos lograr que éste sea un entorno inclusivo. Para ello necesitamos detectar y eliminar las barreras sociales que se dan específicamente a través de las creencias y actitudes de los compañeros del alumnado con discapacidad. En este sentido sólo existen algunas investigaciones que han desarrollado instrumentos para el estudio de las actitudes del alumnado universitarios sin discapacidad hacia la inclusión del alumnado con discapacidad en la Educación Superior (Alonso, Navarro, \& Vicente, 2008; Luque, \& Gutiérrez, 2014; Martínez Martín, 2010; Rodríguez Martín, \& Álvarez Arregui, 2013; Rodríguez Martín \& Álvarez Arregui, 2015; Suriá, Ordoñez, \& Martínez, 2015). Y son menos aun, las que han desarrollado instrumentos con el mismo objetivo basados en el modelo de la Teoría de la Conducta Planificada (i.e., Novo Corti, Muñoz Cantero, \& Calvo Porral, 2011), a pesar de que este modelo aporta una visión metodológica más completa sobre el estudio de las actitudes que otros modelos (Arias et al., 2013). El problema de estos instrumentos y otros construidos 'ad hoc' es que en muchas ocasiones adolecen de limitaciones y defectos metodológicos como la utilización de 
muestras sesgadas y demasiado pequeñas, pruebas de contraste no paramétricas, no incluyen el tamaño del efecto en pruebas de contraste tales como t o ANOVA, entre otros, lo que procura escasas propiedades de fiabilidad y validez en los instrumentos desarrollados.

Por tanto, la principal novedad de nuestra investigación radica en el estudio de las actitudes y la predisposición del alumnado universitario hacia la inclusión del alumnado con discapacidad en la Educación Superior según el modelo de la Teoría de la Conducta Planificada. Siendo escasas las investigaciones en esta misma línea (Kudláček, Sherrill, \& Válková, 2002; Kudláček, 2007; Novo Corti et al., 2012; Novo Corti, et al, 2011; Novo Corti et al., 2015). Este modelo, el de la Teoría de la Conducta Planificada, es uno de los que más amplia repercusión tiene en la investigación sobre las actitudes. Desde esta perspectiva se asume que la mayor parte de la conducta está bajo el control de la persona y, en consecuencia, que el factor fundamental para predecir el comportamiento manifiesto será la intención o motivación deliberada para actuar (Arias et al., 2013). A su vez, esta variable, la intención del comportamiento, está directamente influida por las siguientes variables: actitudes, normas subjetivas, y el control del comportamiento percibido. Mientras que las actitudes están formadas por la propia evaluación que tiene la persona de ella misma hacia un comportamiento objetivo, las normas subjetivas se relacionan con la percepción que tiene la persona hacia la presión social a la hora de realizar el comportamiento. Por tanto, este modelo ofrece un análisis y visión más completa de la evaluación de las actitudes. Además añade Novo Corti et al., (2012) que este modelo ofrece una alternativa a la valoración de las actitudes mediante análisis factoriales como los de Akrami, Ekehammar, Claesson, \& Sonnander, (2006) o Scior \& Furnham, (2011). Por tanto, consideramos relevante basarnos en este modelo para nuestra investigación, en la que se destaca como determinante del comportamiento la intención (Steinmetz, Knappstein, Ajzen, Schmidt, \& Kabst, 2016), centrándonos en los estudiantes universitarios en cuanto a la conducta de incluir a compañeros con discapacidad en la universidad.

\section{Objetivo}

De este modo, nuestro trabajo surge como una propuesta para responder a estas necesidades, entre las que destaca el contar con instrumentos objetivos, fiables y válidos para evaluar las actitudes de los estudiantes universitarios hacia el alumnado con discapacidad dentro del marco de la Educación Superior, siguiendo el modelo de la Teoría de la Conducta Planificada. Por tanto, el objetivo general consiste en desarrollar un instrumento con el máximo ri- 
gor metodológico para aportar evidencias de validez y fiabilidad, que permita evaluar las actitudes sobre la conducta de incluir al alumnado universitario con discapacidad por parte de los estudiantes universitarios.

\section{Método}

\section{Participantes}

En este estudio participaron un total de 1044 universitarios/as de diferentes carreras y niveles, de los cuales 623 estudian en universidades españolas y 421 en universidades argentinas. Los criterios de inclusión fueron: a) Ser estudiante de alguna universidad española o argentina, y b) No tener discapacidad. En cuanto a la distribución según el género con el que se identifican, se reveló una mayor representación de mujeres $(n=762 ; 73 \%)$ que de hombres ( $\mathrm{n}=275 ; 26 \%)$ y que de personas con identidades transgénero $(\mathrm{n}=3 ; 0,29 \%)$. El hecho de que exista un mayor porcentaje de mujeres participantes, consideramos que puede ser debido a las titulaciones a las que hemos tenido acceso en las que suele predominar el género femenino (e.g., Educación Infantil, Enfermería, Trabajo Social, etc.). De acuerdo a las edades, oscilaron entre los 17 y los 64 años, situándose la media en 23,51 años (DT = 6,34). Si analizamos los porcentajes más significativos, observamos que el $61,5 \%$ de los participantes tienen edades comprendidas entre los 19 y los 23 años.

Asimismo, el mayor porcentaje de participantes de esta investigación, estudian titulaciones relacionadas con Educación (Magisterio en Educación Infantil, en Educación Primaria y Educación Social) con un 24,7\% ( $\mathrm{n}=258)$, seguido de Trabajo Social con un $14,8 \%$ ( $\mathrm{n}=$ 154), Derecho o Abogacía con un 14,1\% ( $\mathrm{n}=147)$, estudiantes para Profesorado de diferentes especialidades con 10,3\% ( $\mathrm{n}=108)$ e Ingeniería y Arquitectura con 10,3\% ( $\mathrm{n}=108)$, como puede apreciarse en la Tabla 1 que aparece a continuación. 
Tabla 1. Titulaciones agrupadas según conocimiento que cursa el alumnado participante de las universidades españolas y argentinas

\begin{tabular}{|c|c|c|}
\hline Titulaciones agrupadas & $\mathbf{N}$ & $\%$ \\
\hline Educación & 258 & 24,7 \\
\hline Trabajo Social & 154 & 14,8 \\
\hline Derecho - Abogacía & 147 & 14,1 \\
\hline Ciencias de la Salud & 146 & 14 \\
\hline Profesorado & 108 & 10,3 \\
\hline Ingeniería y Arquitectura & 108 & 10,3 \\
\hline Pedagogía y Psicopedagogía & 26 & 2,5 \\
\hline Administración y Economía & 25 & 2,4 \\
\hline Ciencias Políticas & 15 & 1,4 \\
\hline Artes y Humanidades & 14 & 1,3 \\
\hline Ciencias Comunicación & 13 & 1,2 \\
\hline Ciencias Sociales & 10 & 1 \\
\hline Ambientales y Ecología & 5 & 0,5 \\
\hline Perdidos & 15 & 1,4 \\
\hline Total & 1044 & 100 \\
\hline
\end{tabular}

\section{Instrumento}

El instrumento utilizado fue la escala desarrollada siguiendo el marco teórico de la Teoría de la Conducta Planificada. El proceso de construcción de esta escala fue minucioso con el que se trató de asegurar la validez de contenido a través de la aplicación de cuatro criterios: la revisión de la literatura científica, la concordancia entre jueces expertos, la validación y reformulación de los ítems según los resultados de la prueba piloto y del grupo de discusión y, finalmente, el análisis del poder discriminativo de los ítems (prueba Kruskal-Wallis $y \chi 2)$.

La consulta a los jueces expertos se llevó a cabo entre septiembre de 2013 y marzo de 2014, en la que participaron ocho profesores/as de la Universidad de Valladolid y de la Universidad de Salamanca, algunos de ellos miembros del Instituto Universitario de Integración en la Comunidad, INICO), expertos en Psicología, Pedagogía y Discapacidad. De la evaluación realizada por los jueces se extrajo la escala piloto que se probó en un grupo de 57 participantes para comprobar la comprensión de los ítems por parte del alumnado. De los resultados extraídos, se evidenció la necesidad de modificar la redacción de algunos de los ítems. Para ello se realizó un grupo de discusión con expertos que nos hicieron apreciaciones concretas para su reformulación. De esta manera obtuvimos la versión de la escala con la que recogimos los datos de la muestra final $(\mathrm{N}=1044)$. Esta escala consta de 41 ítems (vid. Tabla 2) 
que definen aspectos observables, concretos y entendibles relacionados con la conducta de incluir a compañeros con discapacidad en la universidad. Están enunciados con formato declarativo en primera persona y ordenados en torno a las cuatro dimensiones (Actitudes, Normas Subjetivas, Control del Comportamiento Percibido e Intención) que sustentan el constructo teórico de la Teoría de la Conducta Planificada y con un formato de respuesta escala Likert de seis puntos (evitando así la tendencia central) para cada una de estas dimensiones:

1) Intención: 1. Totalmente en desacuerdo (TDS); 2. Bastante en desacuerdo (BD); 3. Algo en desacuerdo (AD); 4. Algo de acuerdo (AA); 5. Bastante de acuerdo (BA); y 6. Totalmente de acuerdo (TA).

2) Actitudes: 1. Totalmente improbable (TI); 2. Bastante improbable (BI); 3. Algo improbable (AI); 4. Algo probable (AP); 5. Bastante probable (BP); 6. Totalmente probable (TP).

3) Normas Subjetivas: 1. Nada (N); 2. Casi nada (CN); 3. Poco (P); 4. Algo (A); 5. Bastante (B); 6. Mucho (M).

4) Control del Comportamiento Percibido: 1. Totalmente en desacuerdo (TDS); 2. Bastante en desacuerdo (BD); 3. Algo en desacuerdo (AD); 4. Algo de acuerdo (AA); 5. Bastante de acuerdo (BA); 6. Totalmente de acuerdo (TA).

Tabla 2. Escala de Actitudes hacia la inclusión de las personas con discapacidad en la Educación Superior

\section{Intención}

I1. Me gustaría que mi universidad realizara programas de sensibilización para mejorar la inclusión de las personas con discapacidad en la universidad.

I2. Quiero participar en programas de apoyo a estudiantes con discapacidad en la universidad.

I3. Me gustaría trabajar con un/a compañero/a con discapacidad durante el periodo de estudios universitarios.

I4. Si tuviera compañeros/as con discapacidad, les ayudaría cuando me lo demandasen.

I5. Si tengo amistad con un/a compañero/a con discapacidad, intento estar con él/ella también fuera del entorno universitario.

I6. Promuevo las ventajas de ayudar a las personas con discapacidad entre mi familia y amigos.

I7. Sabría explicar cinco razones por las que es beneficioso incluir a compañeros/as con discapacidad en mi clase.

I8. No me importaría tener compañeros/as con discapacidad en mi clase.

I9. Asisto a programas de formación para aprender sobre temas relacionados con discapacidad.

I10. Si tuviera un/a compañero/a con discapacidad en mi clase, le propondría formar parte de mi equipo.

I11. Me gusta que las personas con discapacidad puedan estudiar en mi universidad.

I12. Si tuviera un compañero/a con discapacidad en mi clase, me gustaría que se sintiera como uno más de la clase.

\section{Actitudes}

A1. A mi familia no le importaría que hiciera trabajos de la carrera con compañeros/as con discapacidad.

A2. La ayuda a las personas con discapacidad es un problema que debe afrontar e intentar resolver la persona y su propia familia.

A3. Si hubiera personas con discapacidad en mi clase, sufrirían discriminación.

A4. Si se incluyera a personas con discapacidad en mi clase, se reduciría la calidad de las clases.

A5. Es una pérdida de tiempo ayudar a las personas con discapacidad.

A6. Estoy a favor de que el alumnado con discapacidad tenga oportunidades de estudiar mi carrera. 

A7. La inclusión de un/a compañero/a con discapacidad en mi clase es una oportunidad para entablar una nueva
amistad.
A8. Las ventajas de incluir a personas con discapacidad en la universidad superan a las desventajas.
A9. Me preocupa que las personas con discapacidad tengan dificultades para acceder a mi carrera universitaria.
A10. Si un/a compañero/a con discapacidad necesitase ayuda, sería importante que yo le ayudara
A11. Me preocupa no saber cómo incluir a un/a compañero/a con discapacidad en clase.

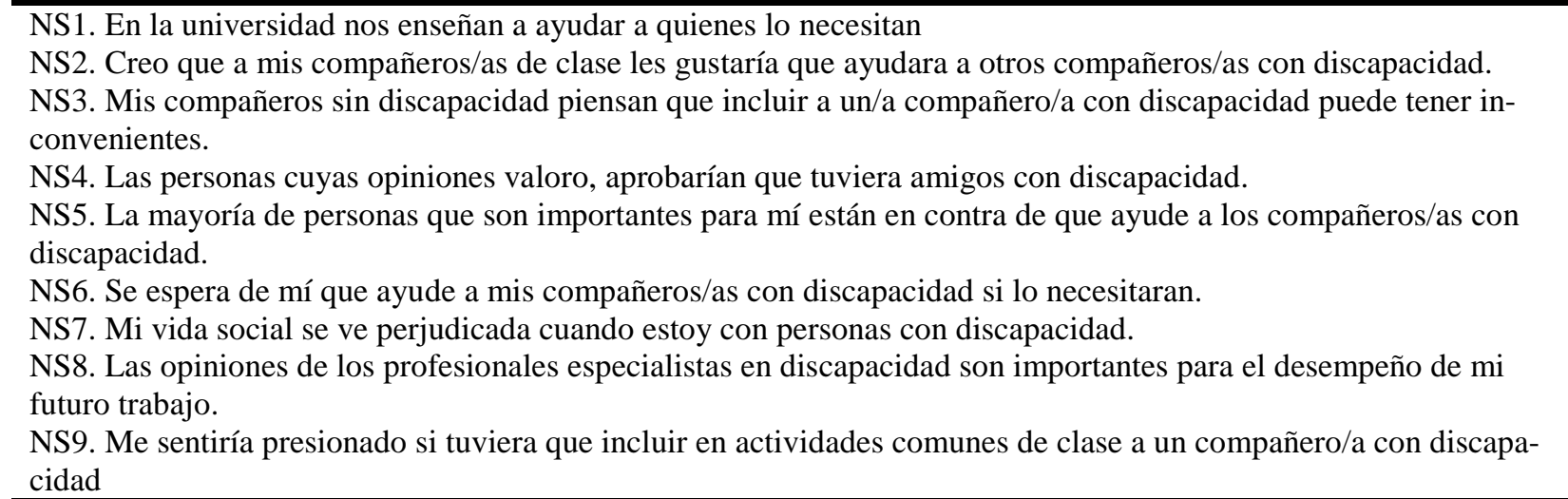

\section{Control del Comportamiento Percibido}

CCP1. Para mí es fácil incluir a personas con discapacidad en mi clase cuando alguien me explica cómo tengo que hacerlo.

CCP2. Mi universidad tiene los recursos para que haya una inclusión eficaz de estudiantes con discapacidad.

CCP3. Si tuviéramos a un compañero/a con discapacidad, nos informarían sobre su discapacidad.

CCP4. Estoy seguro/a de que si quiero puedo ayudar a mis compañeros/as con discapacidad.

CCP5. La decisión de incluir a personas con discapacidad en mis trabajos de clase dependería de otras personas. CCP6. Tengo los recursos, los conocimientos y la capacidad para poder incluir a las personas con discapacidad en mi clase.

CCP7. Entiendo lo que es la inclusión de las personas con discapacidad, tanto en la teoría como en la práctica.

CCP8. Es difícil incluir a compañeros con discapacidad en la universidad.

CCP9. Si tuviera un/a compañero/a con discapacidad y necesitara ayuda, me gustaría ayudarle pero no sabría cómo hacerlo

Estas cuatro dimensiones tratan de explicar y predecir una conducta, que en este caso es la de incluir a personas con discapacidad en la universidad (vid. Figura 1). De acuerdo con Ajzen, $(2011,2012)$ y la TPB, las acciones humanas están influidas principalmente por tres factores: Una evaluación favorable o desfavorable hacia el comportamiento "Actitudes" (creencias hacia el comportamiento), la presión social percibida para realizar o no una conducta "Normas subjetivas" (creencias del individuo sobre las normas sociales o lo que piensan los otros de ellas) y la capacidad percibida para realizar una conducta o el "Control del Comportamiento Percibido" (las creencias que tiene la persona sobre la dificultad o facilidad para llevar a cabo un comportamiento determinado) (Ajzen, 2006; Ajzen \& Fishbein, 2005). La combinación de estas variables (las actitudes, las normas subjetivas y el control del comportamiento percibido) originan la formación de la intención de realizar un comportamiento y, por tanto, el comportamiento. De esta manera, podremos predecir los comportamientos relacionados con la inclusión de las personas con discapacidad. 


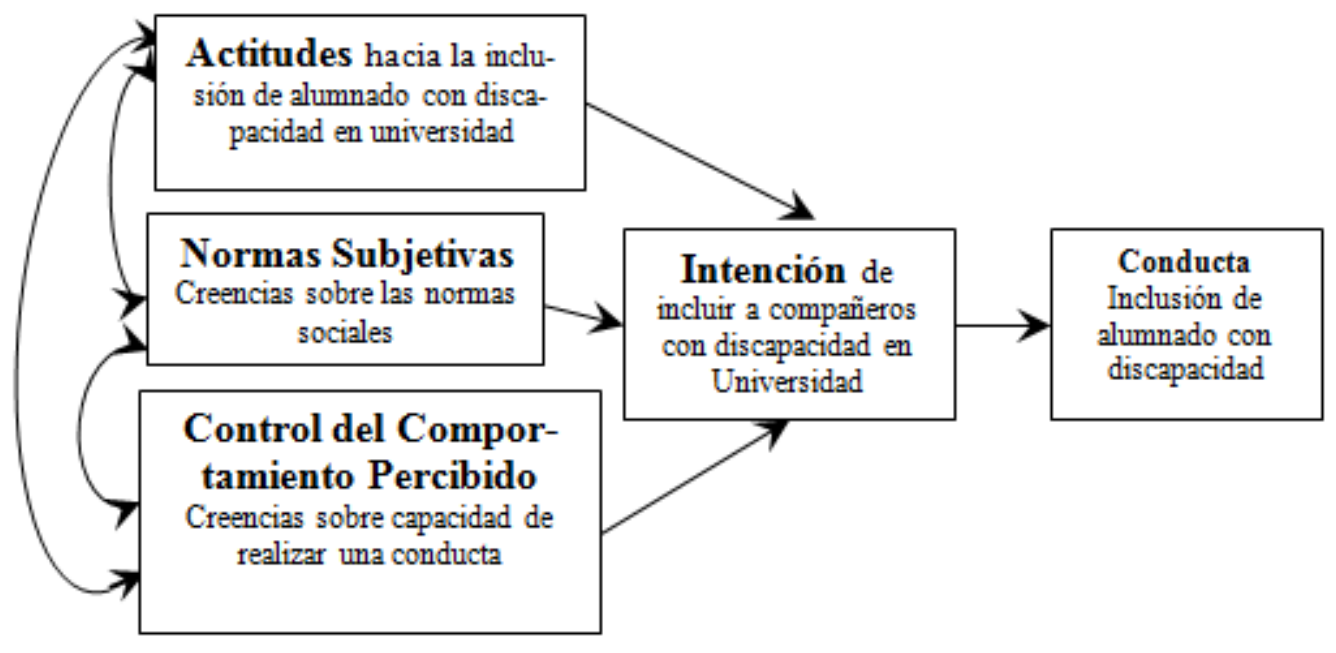

Figura 1. Modelo de la Teoría de la Conducta Planificada con el objeto de este estudio (adaptado de Ajzen, 2006)

Estos ítems de la escala (que mostramos anteriormente en la Tabla 2) vienen precedidos por unas breves instrucciones y un apartado donde se recogen datos referidos a las siguientes variables: Edad, Sexo/Género (1. Hombre, 2. Mujer, y 3. Otro (identidades transgénero), Estudios que cursa actualmente (Carrera y año). Además de recoger esta información, también les preguntamos sobre si conocen a personas con discapacidad: ¿Tiene algún tipo de contacto con personas con discapacidad? (1. Sí, 2. No); En caso de tener contacto con personas con discapacidad señalar la razón (1. Familiar; 2. Compañero/a de estudios; 3. Laboral; 4. Asistencial; 5. Ocio/amistad; 6. Otras razones), la frecuencia (1. Casi permanente, 2. Habitual, 3. Frecuente, 4. Esporádica) y el tipo de discapacidad de las personas con las que tiene contacto (1. Física, 2. Auditiva, 3. Visual, 4. Intelectual, 5. Múltiple).

\section{Procedimiento}

La selección del alumnado universitario, tanto en España como en Argentina, se realizó a través de un muestreo no probabilístico incidental (muestra de conveniencia) dada la imposibilidad de realizar un muestreo aleatorio en la práctica. Contactamos con el profesorado universitario al que teníamos acceso, y con las personas que aceptaron a colaborar aplicamos la escala a su alumnado tanto de forma presencial como online. En los casos en los que la aplicación fue presencial, estuvo presente la investigadora y se les proporcionaron las copias necesarias de la escala en papel. Por otro lado, en los que casos en los que la aplicación fue online se les proporcionó un enlace a la escala virtual desarrollada en el programa LimeSur- 
vey. Posteriormente, se utilizó la estrategia bola de nieve ya que fueron animándose unas personas a otras a participar en la investigación. Por este motivo, no podemos afirmar que se haya realizado un "muestreo" en el sentido estricto de la palabra sino que el criterio tomado fue el acceso y la disponibilidad de las personas participantes (i.e., alumnado universitario sin discapacidad de los centros). Asimismo, teniendo en cuenta las características de las personas que queríamos evaluar (personas mayores de 18 años), no ha sido necesario solicitar autorización al Comité de Bioética de la Universidad de Valladolid ni de los Comités de Bioética de las Universidades Argentinas. Se garantizó la confidencialidad de los datos y el uso exclusivo de los mismos con fines de investigación.

\section{Análisis de datos}

El análisis de los datos se realizó mediante el paquete estadístico SPSS 15 y LISREL 8.8. El método que empleamos para analizar la fiabilidad y validez constructo (estructura interna de la escala) fue el Análisis Factorial Confirmatorio (AFC), que se ha convertido en uno de los procedimientos más utilizados en investigación en ciencias sociales (Arias, 2008). El $A F C$ es un procedimiento de análisis encuadrado en los modelos de ecuaciones estructurales (SEM, Structural Equation Modeling). Aplicamos estos procedimientos porque nos permiten conocer el grado en que las relaciones entre los ítems se corresponden con el constructo que pretendemos evaluar (Gómez, 2010), es decir, contrastar en qué medida un conjunto de indicadores se relacionan con una o más variables latentes (observables) o factores y apoyan un modelo teórico (Arias, 2008).

\section{Resultados}

\section{Análisis de la fiabilidad y validez del instrumento}

El procedimiento del Análisis Factorial Confirmatorio se ha aplicado a los datos que recogimos del alumnado universitario $(\mathrm{N}=1044)$ con la Escala. Estas respuestas nos han permitido contrastar si el instrumento es válido y fiable para medir la intención del alumnado universitario de incluir a personas con discapacidad en la universidad. Antes de comenzar con el procedimiento del $A F C$ propiamente dicho, comprobamos a través de la prueba de adecuación muestral de Kaiser-Meyer-Olkin (KMO) y la prueba de esfericidad de Barlett que todos los índices de los ítems en su conjunto nos indicaran la idoneidad de llevar a cabo el Análisis Factorial. Los resultados que obtuvimos fueron de un índice de $K M O$ en el total de la escala de ,893 y una significación (p) en todos los factores de $\mathrm{p} \leq, 001$. Asimismo, se realiza- 
ron análisis descriptivos de los ítems (vid. tabla 3) y se obtuvieron índices de asimetría (“Asi”) y curtosis (“Cu”) próximos a cero y por debajo del valor 2, lo que índica semejanza a la curva normal (se cumplieron en la mayoría de los casos excepto en la curtosis de Intención = 4,46 y de Actitudes = 5,03).

Tabla 3. Estadísticos descriptivos de las puntuaciones de la Escala.

\begin{tabular}{cccccccccccccccccc}
\hline \multicolumn{11}{c}{ Descriptivos } \\
\hline DIM & $N$ & $M$ & $E T M$ & $M d n$ & Mod & $M n$ & $M x$ & $P M T$ & Var & $D T$ & $R n$ & $P$ & $P$ & $P$ & $A s i$ & $C u$ \\
INT & 988 & 58,99 & 0,27 & 60 & 62 & 12 & 72 & 36 & 69,78 & 8,35 & 60 & 55 & 60 & 65 & - & 4,46 \\
ACT & 987 & 44,12 & 0,15 & 44 & 46 & 11 & 61 & 33 & 21,47 & 4,63 & 50 & 42 & 44 & 47 & - & \\
NS & 1004 & 31,14 & 0,14 & 31 & 33 & 13 & 44 & 27 & 19,11 & 4,37 & 31 & 29 & 31 & 34 & - & 0,29 & 0,64 \\
CCP & 1007 & 36,15 & 0,15 & 36 & 37 & 14 & 54 & 27 & 22,18 & 4,71 & 40 & 33 & 36 & 39 & $-0,2$ & 0,99 \\
Total & 893 & 170,42 & 0,48 & 172 & 171 & 100 & 209 & 123 & 205,64 & 14,34 & 109 & 162 & 172 & 181 & - & 0,78 & 1,33 \\
\hline
\end{tabular}

Nota: M = Media, ETM = Error Típico de Medida, Mdn = Mediana, Mod= Moda, Mn = Mínimo, Mx= Máximo, PMT = Punto Medio Teórico, Var = Varianza, DT= Desviación Típica, $\mathrm{Rn}=$ Rango, $\mathrm{P}=$ Percentiles, Asi = Asimetría, $\mathrm{Cu}=\mathrm{Curtosis}$.

En cuanto a las evidencias de validez del instrumento basadas en el contenido, aportamos las pruebas expuestas anteriormente (revisión bibliográfica, análisis de concordancia entre jueces expertos, validación y reformulación según prueba piloto, y grupo de discusión) $\mathrm{y}$, por otro lado, los resultados obtenidos del poder discriminativo de los ítems de la prueba no paramétrica Kruskal-Wallis y el $\chi 2$ (cuanto más grande sea este valor más poder discriminativo tiene el ítem) que avalan la utilidad de la Escala para evaluar la intención del alumnado universitario de incluir a personas con discapacidad en la Universidad. Nuestro objetivo al analizar el poder discriminativo de los ítems fue determinar si éstos permitían discriminar entre grupos previamente establecidos (aquellos derivados de tomar como punto de corte los $C_{25}$ y $C_{75}$ ) en cada una de las cuatro dimensiones (Intención, Actitudes, Normas Subjetivas y Control del Comportamiento Percibido) y en la puntuación total de la Escala. De los resultados obtenidos destacamos que todos los contrastes resultaron significativos $(p=, 000)$ en todos los ítems, en cada una de las dimensiones y en el total de la escala. De este modo concluimos que todos los ítems tienen poder discriminativo entre los grupos de personas con puntuaciones bajas, medias y altas. 
Con relación a las evidencias aportadas sobre la validez de constructo basada en la estructura interna de la Escala, estudiamos los resultados obtenidos de las pruebas realizadas mediante el Análisis Factorial Confirmatorio (AFC). Por un lado estudiamos el "Modelo 1" siguiendo la Teoría de la Conducta Planificada en la que se presentan tres factores latentes (Actitudes, Normas Subjetivas, Control del Comportamiento Percibido) que componen a su vez el factor Intención, constituyéndose estas cuatro variables con un total de 41. Los resultados que encontramos en el Modelo 1 (vid. tabla 4) fueron que en algunas dimensiones la precisión predictiva no era adecuada al constructo que se pretende medir, ni existía un buen ajuste al modelo. Tras realizar dicha evaluación del modelo, determinaremos que era preciso realizar modificaciones en el mismo.

Tabla 4. Cargas factoriales ( $\lambda)$, coeficientes de determinación $\left(R^{2}\right)$, errores de predicción ( $\theta)$ y valores de t, en relación a los ítems con las dimensiones Normas Subjetivas y Control del Comportamiento Percibido del Modelo 1

\begin{tabular}{|c|c|c|c|c|c|c|c|c|c|}
\hline \multicolumn{10}{|c|}{ Estimación de los parámetros del Modelo 1} \\
\hline \multicolumn{5}{|c|}{ Intención } & \multicolumn{5}{|c|}{ Actitudes } \\
\hline Ítem & $\lambda$ & $\mathrm{R}^{2}$ & $\Theta$ & $\mathrm{T}$ & Ítem & $\lambda$ & $\mathrm{R}^{2}$ & $\Theta$ & $\mathrm{t}$ \\
\hline Int01 & 0,08 & 0,01 & 0,99 & & Act01 & 0,05 & 0 & 1 & 1,34 \\
\hline Int02 & 0,01 & 0 & 1 & 0,24 & Act02 & 0,02 & 0 & 1 & 0,73 \\
\hline Int03 & 0,53 & 0,28 & 0,72 & 2,26 & Act03 & 0,26 & 0,07 & 0,93 & 7,81 \\
\hline Int04 & 0,56 & 0,31 & 0,69 & 2,27 & Act04 & 0,38 & 0,15 & 0,85 & 11,77 \\
\hline Int05 & 0,59 & 0,35 & 0,65 & 2,27 & Act05 & 0,64 & 0,41 & 0,59 & 21,27 \\
\hline Int06 & 0,33 & 0,11 & 0,89 & 2,22 & Act06 & 0,57 & 0,33 & 0,67 & 18,39 \\
\hline Int07 & 0,22 & 0,05 & 0,95 & 2,14 & Act07 & 0,65 & 0,43 & 0,57 & 21,70 \\
\hline Int08 & 0,08 & 0,01 & 0,99 & 1,62 & Act08 & 0,6 & 0,36 & 0,64 & 19,69 \\
\hline Int09 & 0,05 & 0 & 1 & 1,19 & Act09 & 0,48 & 0,23 & 0,77 & 14,88 \\
\hline int 10 & 0,65 & 0,43 & 0,57 & 2,27 & Act 10 & 0,55 & 0,30 & 0,70 & 17,59 \\
\hline int11 & 0,01 & 0 & 1 & 0,39 & Act11 & 0,76 & 0,58 & 0,42 & 26,45 \\
\hline int 12 & 0 & 0 & 1 & 0,11 & & & & & \\
\hline \multicolumn{5}{|c|}{ Normas Subjetivas } & \multicolumn{5}{|c|}{ Control Comportamiento Percibido } \\
\hline Ítem & $\lambda$ & $\mathrm{R}^{2}$ & $\Theta$ & $\mathrm{T}$ & Ítem & $\lambda$ & $\mathrm{R}^{2}$ & $\theta$ & $\mathrm{t}$ \\
\hline$\overline{N s 01}$ & 0,9 & 0,81 & 0,19 & 36,04 & Ccp01 & 0,49 & 0,24 & 0,76 & 15,82 \\
\hline $\mathrm{Ns} O 2$ & 0,59 & 0,35 & 0,65 & 20,08 & Сср02 & 0,36 & 0,13 & 0,87 & 11,12 \\
\hline Ns03 & 0,40 & 0,16 & 0,84 & 12,77 & Сср03 & 0,33 & 0,11 & 0,89 & 10,21 \\
\hline Ns04 & 0,57 & 0,33 & 0,67 & 19,3 & Ccp04 & 0,69 & 0,47 & 0,53 & 23,98 \\
\hline Ns05 & 0,59 & 0,34 & 0,66 & 19,89 & Ccp05 & 0,38 & 0,14 & 0,86 & 11,96 \\
\hline Ns06 & 0,66 & 0,43 & 0,57 & 22,88 & Сср06 & 0,41 & 0,17 & 0,83 & 12,86 \\
\hline Ns07 & 0,58 & 0,33 & 0,67 & 19,49 & Сср07 & 0,72 & 0,52 & 0,48 & 25,47 \\
\hline Ns08 & 0,55 & 0,30 & 0,70 & 18,26 & Сср08 & 0,54 & 0,29 & 0,71 & 17,75 \\
\hline $\mathrm{Ns} 09$ & 0,68 & 0,46 & 0,54 & 23,92 & Сср09 & 0,85 & 0,72 & 0,28 & 32,27 \\
\hline
\end{tabular}


De esta forma, realizamos modificaciones sobre la estructura factorial y estimamos el

\section{Modelo 2:}

- La dimensión "Intención" es explicada por los indicadores int_03, int_04, int_05, int_06, int_07e int_10.

- La dimensión “Actitudes" es explicada por los indicadores act_04, act_05, act_06, act_07, act_08, act_09, act_10 y act_11.

- La dimensión "Normas subjetivas" es explicada por los indicadores $n s_{-} 01, n s_{-} 02$, ns_03, ns_04, ns_05, ns_06, ns_07, ns_08, ns_09, ccp_01 ("Para mí es fácil incluir a personas con discapacidad en mi clase cuando alguien me explica cómo tengo que hacerlo) y ccp_06 ("Tengo los recursos, los conocimientos y la capacidad para poder incluir a las personas con discapacidad en mi clase”).

- La dimensión "Control del Comportamiento Percibido" es explicada por los indicadores $c c p \_01, c c p \_02, c c p \_03, c c p \_04, c c p \_05, c c p \_06, c c p \_07, c c p \_08$ y ccp09.

- La dimensión intención no es explicada por las variables Actitudes, Normas Subjetivas y Control del Comportamiento Percibido porque las cargas factoriales son casi inexistentes $(\beta$ Act. $=, 08 ; \beta N S .=, 09 ; \beta C C P=, 03) \mathrm{y}$, por otro, en los valores de $\mathrm{t}$ que todos están por debajo de 1,96 (tAct. $=1,94$; tNS. $=1,39$; y tCCP $=, 50)$. De esta forma corroboramos que no se cumple la hipótesis de que la variable intención es explicada o depende de las otras variables.

Además de estas variaciones en los ítems, se tuvo en cuenta las covarianzas del error y sus correlaciones en los ítems ccp_01 y ccp_07; int_04 e int_06; ns_01 y ns_05; y ns_03 y ns_05, mejorando así las estadísticas de ajuste y la fiabilidad de los coeficientes.

En cuanto a los resultados en la estimación de los parámetros y la evaluación de la bondad de ajuste del modelo (vid. Tabla 5), observamos que las cargas factoriales $\lambda$ (nos indican la dirección y fuerza de la relación entre el factor y cada indicador) superan en su mayoría el valor de ,50 y todas ellas resultaron estadísticamente significativas con valores superiores a $2,58(\mathrm{p}<, 01)$. En cuanto a los errores de predicción $\theta$ oscilan entre ,17 y,96 (por tanto sus coeficientes de determinación $\mathrm{R}^{2}$ están entre ,049 y ,83). De este modo los resultados son aceptables y muestran un mejor ajuste con este modelo. En cuanto a la bondad de ajuste, los resultados obtenidos en NNFI, CFI e IFI alcanzan valores superiores a ,90, RMSEA de 0,66 y $\chi 2$ de 2531,79 con 452 grados de libertad $(\mathrm{p}=, 00)$. Por tanto, concluimos que el ajuste de nuestros datos al modelo es adecuado. 
Tabla 5. Cargas factoriales $(\lambda)$, coeficientes de determinación $\left(R^{2}\right)$, errores de predicción ( $\theta)$ y valores de $t$, en relación a los ítems con las dimensiones Normas Subjetivas y Control del Comportamiento Percibido del Modelo 2

\begin{tabular}{cccccccccc}
\hline \multicolumn{7}{c}{ Estimación de los parámetros del Modelo 2 } \\
\hline \multicolumn{7}{c}{ Intención } \\
\hline Ítem & $\lambda$ & $\mathrm{R}^{2}$ & $\theta$ & $\mathrm{T}$ & Ítem & $\lambda$ & $\mathrm{R}^{2}$ & $\theta$ & $\mathrm{t}$ \\
\hline Int03 & 0,55 & 0,30 & 0,70 & & Act04 & 0,37 & 0,14 & 0,86 & 11,4 \\
Int04 & 0,49 & 0,24 & 0,76 & 10,74 & Act05 & 0,64 & 0,41 & 0,59 & 21,24 \\
Int05 & 0,61 & 0,37 & 0,63 & 11,96 & Act06 & 0,55 & 0,33 & 0,67 & 18,43 \\
Int06 & 0,22 & 0,05 & 0,96 & 5,56 & Act07 & 0,65 & 0,43 & 0,57 & 21,71 \\
Int07 & 0,23 & 0,05 & 0,95 & 5,95 & Act08 & 0,6 & 0,36 & 0,64 & 19,68 \\
Int10 & 0,68 & 0,46 & 0,54 & 12,24 & Act09 & 0,48 & 0,23 & 0,77 & 14,90 \\
& & & & & Act10 & 0,55 & 0,30 & 0,70 & 17,59 \\
& & & & & Act11 & 0,76 & 0,58 & 0,42 & 26,41 \\
\hline \multicolumn{1}{c}{ Normas Subjetivas } & & Control Comportamiento Percibido \\
\hline Item & $\lambda$ & $\mathrm{R}^{2}$ & $\theta$ & $\mathrm{T}$ & Ítem & $\lambda$ & $\mathrm{R}^{2}$ & $\theta$ & $\mathrm{t}$ \\
\hline Ns01 & 0,91 & 0,83 & 0,17 & 36,72 & Ccp01 & 0,51 & 0,2 & 0,8 & 10,67 \\
Ns02 & 0,59 & 0,35 & 0,65 & 20,3 & Ccp02 & 0,36 & 0,13 & 0,87 & 11,25 \\
Ns03 & 0,38 & 0,15 & 0,85 & 12,32 & Ccp03 & 0,33 & 0,11 & 0,89 & 10,33 \\
Ns04 & 0,56 & 0,32 & 0,68 & 19,18 & Ccp04 & 0,70 & 0,49 & 0,51 & 24,4 \\
Ns05 & 0,65 & 0,42 & 0,58 & 21,72 & Ccp05 & 0,37 & 0,14 & 0,86 & 11,55 \\
Ns06 & 0,65 & 0,43 & 0,57 & 23,06 & Ccp06 & $-0,09$ & 0,31 & 0,69 & -2 \\
Ns07 & 0,58 & 0,34 & 0,66 & 19,90 & Ccp07 & 0,69 & 0,48 & 0,52 & 23,96 \\
Ns08 & 0,55 & 0,30 & 0,70 & 18,43 & Ccp08 & 0,54 & 0,29 & 0,71 & 17,63 \\
Ns09 & 0,66 & 0,44 & 0,56 & 23,47 & Ccp09 & 0,88 & 0,77 & 0,23 & 33,48 \\
\hline
\end{tabular}

Respecto a los resultados en la fiabilidad compuesta, que nos permite evaluar con qué rigurosidad los indicadores (variables manifiestas) miden la variable latente, en el total del modelo nos indican que el intrumento mide con precisión el constructo $(\mathrm{pc}=933)$. En cuanto a la consistencia interna obtenemos un índice $\alpha$ de Cronbach $=, 72$ siendo adecuado como exponen Nunnally, \& Bernstein, (1994). Por otro lado, analizando los coeficientes de correlación entre las variables latentes del "Modelo 2" (vid. Tabla 6), los resultados muestran que todas las correlaciones $(r)$ son adecuadas porque están comprendidas entre ,09 y ,47 con relación al contenido del par de constructos latentes. Esto significa que cada variable mide un constructo diferente y que no existe multicolinealidad entre ellas, por lo que estos índices nos aportan más evidencias sobre la validez de la escala. También es importante destacar que las variables que comparten más correlación son Intención y Actitudes $(r=, 47)$, como cabría esperar, y las que menos son Normas Subjetivas e Intención con una $r=, 09$. 
Tabla 6. Coeficientes de correlación entre las variables latentes del Modelo 2

\begin{tabular}{ccccc}
\hline & $A C T$ & $N S$ & $C C P$ & $I N T$ \\
\hline$I N T$ & 0,47 & 0,09 & 0,21 & 1,00 \\
$A C T$ & 1 & 0,16 & 0,15 & 0,47 \\
$N S$ & 0,16 & 1,00 & 0,28 & 0,09 \\
$C C P$ & 0,15 & 0,28 & 1,00 & 0,21 \\
\hline
\end{tabular}

Análisis de las puntuaciones en las dimensiones según las variables sociodemográficas

Las puntuaciones obtenidas de las respuestas del alumnado universitario sin discapacidad participante se analizaron mediante: estadísticos descriptivos, diferencia de medias y Análisis de Varianza (ANOVA). La distribución de las puntuaciones de los participantes en torno a las cuatro dimensiones de la escala, corrobora que los resultados de los estudiantes universitario hacia la inclusión del alumnado con discapacidad en la universidad son positivos (distribuciones con asimetrías negativas que llevan a rechazar la hipótesis de normalidad; la mayoría de medianas están entre 5 y 6 para los ítems de valencia positiva y de 1 , y 2 para los de valencia negativa; las medias, modas y medianas de los ítems están por encima del punto medio teórico; etc.). Si analizamos las respuestas según el "género", observamos que existen diferencias significativas a favor de las mujeres en las puntuaciones de casi todas las dimensiones (Normas Subjetivas, Control del Comportamiento Percibido e Intención) menos en la dimensión Actitudes ( $t=-1,6 ; p=, 100 ; d=978$ ), con resultados en el grupo de mujeres de $M$ $=44,2$ y $D T=4,7$ y en el grupo de hombres $M=43,7$ y $D T=4,4$.

En relación a la "edad", el análisis de la varianza puso de manifiesto que existían diferencias significativas entre los distintos grupos de edad en todas las dimensiones de la escala excepto en Actitudes. En contraposición, con la prueba post hoc de Scheffe no se obtienen diferencias significativas en las dimensiones en función de los grupos de edad.

En cuanto a la "nacionalidad" (española o argentina), observamos que existen diferencias significativas en todas las dimensiones. El alumnado de nacionalidad española obtiene puntuaciones ligeramente superiores en Normas Subjetivas y Control del Comportamiento Percibido y el alumnado de nacionalidad argentina, en Actitudes e Intención. 
Según la "titulación universitaria" del alumnado participante, los resultados de la prueba de F de Levene en las dimensiones corroboran que no se cumple el supuesto de homocedasticidad o igualdad de varianzas. De esta forma empleamos las pruebas robustas de Welch y Brown-Forsythe con las que observamos que existen diferencias significativas en todas las dimensiones según los grupos formados. Posteriormente, aplicamos la prueba post hoc Games - Howell con las que podemos hacer un estudio más concreto de cuáles son los grupos según las titulaciones y dónde se dan estas diferencias dentro de cada dimensión. Encontramos que en la dimensión que se establecen más diferencias significativas entre los grupos, es en la dimensión Normas Subjetivas (20), seguida por la dimensión Intención (11), Control del Comportamiento Percibido (4) y, por último, Actitudes (3). En la dimensión Control del Comportamiento Percibido destaca el hecho de que existan diferencias significativas entre "Educación” y “Trabajo Social” por un lado, y por otro "Educación” y "Profesorado", ya que estas áreas de conocimiento a priori están relacionadas. Las titulaciones que alcanzaron una mediana mayor fueron:

- En la dimensión Actitudes, "Artes y Humanidades" (Me =49; $M=47,8)$, “Ciencias Sociales" $(M e=48 ; M=47,3)$ y “Ciencias de la Comunicación” $(M e=47,8 ; M=46,9)$.

- En la dimensión Normas Subjetivas, "Pedagogía y Psicopedagogía” (Me = 33; $M=$ 33,3), “Educación” (Me =33; $M=33,1)$, “Ciencias de la Salud” $(M e=33 ; M=32,7)$ y “Trabajo Social” $(M e=33 ; M=32,4)$.

- En la dimensión Control del Comportamiento Percibido, “Artes y Humanidades” (Me = 39; $M=36,4)$, "Ciencias de la Comunicación" $(M e=38,5 ; M=38,3)$ y "Educación" $(M e=38 ; M=37,4)$.

- En la dimensión Intención, "Pedagogía y Psicopedagogía” $(M e=66 ; M=65,1)$, “Ciencias Sociales" $(M e=64 ; M=55,8)$, “Trabajo Social” $(M e=63 ; M=61,3) \mathrm{y}$ “Artes y Humanidades" $(M e=62 ; M=59,4)$.

En relación a la variable "contacto con personas con discapacidad", solamente existen diferencias significativas a favor del grupo de alumnos sin discapacidad que sí tienen contacto con personas con discapacidad en la dimensión Intención. En el resto de dimensiones no existen diferencias entre ambos grupos. 


\section{Discusión y conclusiones}

En la literatura reciente la medición de las actitudes es un tema de estudio que ha cobrado relevancia en los últimos años (Araya Cortés, González Arias \& Cerpa Reyes, 2014; Rodríguez Martín \& Álvarez Arregui, 2015; Salinas, 2014; Suriá, Villegas, \& Rosser, 2016). Esto se debe a que es considerado como uno de los factores más influyentes en la inclusión educativa y social de las personas con discapacidad. De este modo, con nuestra investigación contribuimos a analizar las actitudes del alumnado universitario, lo que nos permite tomar conciencia sobre las creencias en torno a la inclusión del alumnado con discapacidad y así poder diseñar programas acordes a trabajar estos aspectos concretos o evaluar programas que ya se estén aplicando para comprobar su eficacia.

De los resultados obtenidos de la aplicación de la escala a los 1044 estudiantes sin discapacidad de universidades españolas y argentinas, y del Análisis Factorial Confirmatorio, se han obtenidos índices satisfactorios en fiabilidad y validez. Primeramente, se han aportado evidencias que avalan la validez del instrumento basadas en el contenido (e.g., exhaustiva revisión bibliográfica, análisis de concordancia entre jueces expertos, validación y formulación de ítems según resultados de grupos de discusión, etc.). Asimismo, el poder discriminativo de los ítems se comprobó mediante la prueba no paramétrica Kruskal-Wallis con unos resultados en los que todos los contrastes fueron significativos $(p=, 000)$.

Con relación a las evidencias aportadas sobre la validez de constructo basada en la estructuta interna de la Escala, por un lado estudiamos el "Modelo 1" del que no no se obtuvieron buenos ajustes al modelo ni parámetros adecuados, por lo que fue modificado y se constituyó el denominado "Modelo 2". Con este segundo modelo se obtienen índices de ajuste adecuados con valores superiores a ,90 en NNFI, CFI e IFI y RMSEA de ,66 y $\chi 2$ de 2531,79 con 452 grados de libertad $(\mathrm{p}=, 00)$. Además, los análisis sobre la fiabilidad compuesta fueron buenos $(\mathrm{pc}=933)$. De estos análisis también extrajimos otra conclusión consistente en que existe muy poca influencia de las variables latentes Actitudes $(\beta=, 08)$, Normas subjetivas ( $\beta$ $=, 09)$ y Control del Comportamiento Percibido $(\beta=, 03)$ sobre la variable Intención, y que por tanto, esta última no depende de las otras tres. Novocorti et al., (2011) coincide con nuestros resultados en que la variable actitudes tiene escasa importancia como variable explicativa de la intención $\left(\beta_{1}=, 07\right)$ aunque en su caso el resto de variables sí que influyen con $\beta_{2}=, 43$ (Normas Subjetivas) y $\beta_{3}=, 23$ (Control del Comportamiento Percibido). En una investiga- 
ción posterior, Novo-corti et al., (2015), obtienen que la influencia de las variables latentes sobre la variable Intención es en Actitud de $\beta 1=.174$, en Normas Subjetivas es de $\beta 3=.236$, y en la variable Control del Comportamiento Percibido es de $\beta 2=.520$. Por tanto esta última variable, el control que tiene la persona sobre su capacidad de apoyar en la inclusión de personas con discapacidad, es la que más influencia tiene sobre la intención pero el resto de variables tampoco tienen gran influencia.

Si observamos los resultados en comparación con las distintas variables sociodemográficas que tuvimos en cuenta en esta investigación, en cuanto al "género" encontramos que no existen diferencias significativas entre hombres y mujeres en las Actitudes como también encontraron Moreno et al., (2006) en su investigación. En el resto de variables (Normas Subjetivas, Control del Comportamiento Percibido e Intención) sí que se encontraron diferencias significativas más positivas en el grupo de las mujeres como también presentan Novocorti et al., (2015). En cuanto a la "nacionalidad" (española o argentina), destacamos el hecho de que existan diferencias significativas en todas las dimensiones. En la población española se dan puntuaciones superiores en las creencias sobre las Normas Sociales (i.e., Normas Subjetivas) y en la percepción que tienen sobre ellos mismos a la hora de llevar a cabo la conducta (i.e., Control del Comportamiento Percibido), y entre la población argentina, se obtienen puntuaciones ligeramente favorables en las creencias sobre la inclusión de las personas con discapacidad (i.e., Actitudes) y la predisposición de incluir a estas personas (i.e., Intención).

Por otro lado, consideramos relevante el análisis de la variable "contacto con personas con discapacidad" porque según la literatura es una de las variables que más influye en la formación de las actitudes (Arias et al., 2013; Suria, 2011). Tanto las experiencias directas como las indirectas hacen que se desarrollen creencias y pensamientos sobre las personas con discapacidad que, a su vez, influyen en los comportamientos. En este sentido encontramos que en nuestro estudio existen diferencias significativas entre las personas que sí han tenido contacto, obtenieno respuestas más favorables en la intención de incluir a personas con discapacidad. Por ende, es importante que en las universidades existan más espacios o programas en los que se fomente tanto el conocimiento, como la información y las relaciones con las personas con discapacidad, y en las que se ofrezca el enfoque de la diversidad como oportunidad. De esta manera, fomentaremos el que cada vez exista una mayor y mejor inclusión. 
En cuanto a las limitaciones de este trabajo, consideramos interesante que se contrasten los resultados obtenidos con la opinión del propio alumnado con discapacidad de las universidades que han participado en la investigación. En este sentido, se da continuidad a esta investigación con el desarrollo de una segunda fase de corte cualitativo que permitirá complementar y comprender con una mayor profundidad el tema investigado. Asimismo, se espera que los resultados que se presentan en este artículo sirvan de guía para futuras investigaciones, considerando fundamental la aplicación y validación de este intrumento en otros contextos universitarios.

\section{Agradecimientos}

Esta investigaciónn ha sido confinanciado por el Fondo Social Europeo y la Junta de Castilla y León, a través de la Consejería de Educación con un contrato de Personal Investigador de Reciente Titulación Universitaria 2011-2015.

\section{Referencias}

Ainscow, M. (1999). Understanding the Development of Inclusive Education. London: Falmer Press. doi:10.4324/9780203016619

Ajzen, I. (2006). Constructing a TPB questionnaire: Conceptual and methodological considerations. Recuperado de: https://people.umass.edu/aizen/pdf/tpb.measurement.pdf

Ajzen, I. (2011). Behavioral interventions: Design and evaluation guided by the theory of planned behavior. En M.M. Mark, S.I. Donaldson, \& B.C. Campbell (Eds.), Social psychology for program and policy evaluation (pp. 74-100). New York: Guilford.

Ajzen, I. (2012). The Theory of Planned Behavior. En P. A. M. Lange, A. W. Kruglanski, \& E. T. Higgins (Eds.), Handbook of theories of social psychology (Vol. 1, pp. 438-459). London, UK.: Sage.

Ajzen, I., \& Fishbein, M. (2005). The influence of attitudes on behaviour. En D. Albarracín, B. T. Johnson (Ed.), The Handbook of Attitudes (pp. 173-221). Mahwah, NJ: Erlbaum.

Akrami, N., Ekehammar, B., Claesson, M., \& Sonnander, K. (2006). Classical and modern prejudice: Attitudes toward people with intellectual disabilities. Research in Developmental Disabilities, 27(6), 605-617. doi:10.1016/j.ridd.2005.07.003

Ali, A., Strydom, A., Hassiotis, A., Williams, R., \& King, M. (2008). A measure of the perceived stigma of intellectual disability. British Journal of Psychiatry, 193(5), 410- 
415. doi:10.1192/bjp.bp.107.045823

Alonso, M., Navarro, R., \& Vicente, L. (2008). Actitudes hacia la diversidad en estudiantes universitarios. Castellón: Universidad Jaume I.

Antonak, R.F. (1982). Development and psychometirc analysis of the Scale of Attitudes Toward Disabled Persons. Journal of Applied Rehabilitation Counseling, 13, 22-29.

Araya Cortés, A., González Arias, M., \& Cerpa Reyes, C. (2014). Actitud de universitarios hacia las personas con discapacidad. Educación y Educadores, 17(2), 289-305. doi:10.5294/edu.2014.17.2.5

Arias, B. (2008). Desarrollo de un ejemplo de análisis factorial confirmatorio con LISREL, AMOS y SAS. En M.A. Verdugo, M. Crespo, M. Badía y B. Arias (Coord.), Metodología en la investigación sobre discapacidad. Introducción al uso de las ecuaciones estructurales (pp. 75-120). Salamanca: INICO.

Arias, B., \& Morentin, R. (2003). Actitudes de los pofesionales hacia la discapacidad en Castilla y León. Valladolid: Junta de Castilla y León.

Arias, B., Verdugo, M.A., Gómez, L.E., \& Arias, V. (2013). Actitudes hacia la inclusión. En M.A. Verdugo \& R.L. Shalock (Ed.), Discapacidad e inclusión: manual para la docencia (pp. 61-88). Salamanca: Amarú.

Barnes, C., \& Mercer, G. (Eds). (2004). Chapter 1. Theorising and Researching Disability from a Social Model Perspective. Implementing the social model of disability: Theory and research. (Vol. 1, pp. 1-17). Leeds (UK): Disability Press.

Barton, L. (2009). Estudios sobre discapacidad y la búsqueda de la inclusividad: Observaciones. Revista de Educación, 349, 137-152.

Booth, T. (1996). A Perspective on Inclusion from England. Cambridge Journal of Education, 26(1), 87-99. doi:10.1080/0305764960260107

Gómez, L.E. (2010). Evaluación de la calidad de vida en los servicios sociales: Validación y calibración de la Escala Gencat (Tesis doctoral). Universidad de Salamanca, Salamanca.

Hatton, C., Akram, Y., Robertson, J., Shah, R., \& Emerson, E. (2003). The disclosure process and its impact south Asian families with a child with severe intellectual disabilities. Journal of Applied Research in Intellectual Disabilities, 16, 177-188. doi:10.1046/j.1468-3148.2003.00167.x

Konur, O. (2006). Teaching disabled students in higher education. Teaching in Higher Education, 11, 351-363. doi:10.1080/13562510600680871

Kudláček, M. (2007). Components of Attitudes Toward Inclusion of Students With Physical Disabilities in Physical Education in the Revised "Atipdpe-R" Instrument/Scale for 
Prospective Czech Educators. Acta Univ. Palacki. Olomuc., Gymn. 37(1), 13-18.

Kudláček, M., Sherrill, C., \& Válková, H. (2002). Components/indicators of attitudes toward inclusion of students with physical disabilities in PE in the ATIPDPE instrument/Scale for prospective Czech physical educators. Acta Universitatis Palackianae Olomucensis. Gymnica, 32(2), 35-39. Recuperado de: https://pdfs.semanticscholar.org/ac81/99d955c929efdebfd34cafdf55ce7dab2b69.pdf

Luque, A., \& Gutiérrez, R. (2014). La integración educativa y social del alumnado con discapacidad en el EEES : Universidad de Bolonia. Revista Complutense de Educación, 25(1), 153-175. doi:http://dx.doi.org/10.5209/rev_RCED.2014.v25.n1.41344

Martínez Martín, M.A. (2010). Los estudiantes con discapacidad en la Universidad de Burgos (Tesis doctoral). Universidad de Burgos, Burgos.

Moreno, J., Rodríguez, I., Saldaña, D., \& Aguilera, A. (2006). Actitudes ante la discapacidad en el alumnado universitario matriculado en materias afines. Revista Iberoamericana de Educación, 40(5), 1-12.

Novo Corti, I., \& Muñoz Cantero, J.M. (2012). Los estudiantes universitarios ante la inclusión de sus compañeros con discapacidad: indicadores basados en la teoría de la acción razonada para los estudios de economía y empresa en la Universidad de A Coruña. Revista Española de Orientación y Psicopedagogía, 23(2), 105-122. doi:10.5944/reop.vol.23.num.2.2012.11452

Novo Corti, I., Muñoz Cantero. J.M., Calvo Porral, C. (2011). Analisis de las actitudes de los jovenes universitarios hacia la discapacidad: Un enfoque desde la teoria de la accion. RELIEVE - Revista Electrónica de Investigación y Evaluación Educativa, 17(2), 1-26. doi:10.7203/relieve.17.2.4010

Novo Corti, I., Muñoz Cantero, J.M, \& Calvo Babío, N. (2015). Los futuros docentes y su actitud hacia la inclusión de personas con discapacidad. Una perspectiva de género. Anales de Psicología, 31, 155-171. doi:http://dx.doi.org/10.6018/analesps.31.1.163631

Nunnally, J.C. \& Bernstein, I.H. (1994). Psychometric theory ( $3^{\text {rd }}$ ed.). New York: McGrawHill.

ONU (2006). Convención sobre los derechos de las personas con discapacidad. New York: Organización de las Naciones Unidas.

Rodríguez Martín, A. \& Álvarez Arregui, E. (2013). Development and validation of a scale to identify attitudes towards disability in Higher Education. Psicothema, 25(3), 370-6. doi: 10.7334/psicothema2013.41.

Rodríguez Martín, A., \& Álvarez Arregui, E. (2015). Universidad y discapacidad: Actitudes 
del profesorado y de estudiantes. Perfiles Educativos, 37(147), 86-102. doi: https://doi.org/10.1016/j.pe.2014.09.001

Salinas, M. (2014). Actitudes de estudiantes sin discapacidad hacia la inclusión de estudiantes con discapacidad en la educación superior (Tesis doctoral). Universitat Autónoma de Barcelona, Barcelona.

Scior, K., \& Furnham, A. (2011). Development and validation of the intellectual disability literacy scale for assessment of knowledge, beliefs and attitudes to intellectual disability. Research in Developmental Disabilities, 32(5), 1530-1541. doi:10.1016/j.ridd.2011.01.044.

Steinmetz, H., Knappstein, M., Ajzen, I., Schmidt, P., \& Kabst, R. (2016). How Effective are Behavior Change Interventions Based on the Theory of Planned Behavior? Zeitschrift Für Psychologie, 224(3), 216-233. doi: 10.1027/2151-2604/a000255

Suriá, R. (2011). Análisis comparativo sobre las actitudes de los estudiantes hacia sus compañeros con discapacidad. Electronic Journal of Research in Educational Psychology, 9(1), 197-216.

Suriá, R., Ordoñez, T. \& Martínez Macía, D. (2015). Validación de la escala actitudinal hacia la discapacidad entre los estudiantes universitarios. En M.T. Tortosa, J.D. Álvarez, N. Pellín (Coords.). XIII Jornadas de Redes de Investigación en Docencia Universitaria: Nuevas estrategias organizativas y metodológicas en la formación universitaria para responder a la necesidad de adaptación y cambio (pp 2612-2621). Alicante: Universidad de Alicante.

Suriá, R., Villegas, E., \& Rosser, A. (2016). Discapacidad e integración en el ámbito universitario. Investigación e Innovación Educativa en Docencia Universitaria: Retos, Propuestas y Acciones, 1034-1044. Recuperado de: https://rua.ua.es/dspace/bitstream/10045/60287/1/Investigacion-e-Innovacion-Educativaen-Docencia-Universitaria_061.pdf

Verdugo, M. A., Arias, B., \& Jenaro, C. (1994). Actitudes hacia las personas con minusvalía. Madrid: IMSERSO.

Verdugo, M. A., Jenaro, C., \& Arias, B. (1995). Actitudes sociales y profesionales hacia las personas con discapacidad: estrategias de evaluación e intervención. En M.A. Verdugo \& A. Aguado (Eds.), Personas con discapacidad: perspectivas psicopedagógicas y rehabilitadoras. (pp. 79-143). México: Siglo XXI.

Yuker, H. E., \& Block, J. R. (1986). Research with the Attitudes Toward Persons Scale (ATDP) 1960-1985. New York: Hofstra University Bookstore. 